\title{
An Adaptive Technique for Regularized Level Set Evolution to Image Segmentation
}

\author{
${ }^{1}$ KusumanchiAvinashkumar, ${ }^{2}$ SatyaSwathiBylapudi \\ ${ }^{I}$ Asst. Professor, Dept. of ECE, DIET, Anakapalle, A.P. \\ ${ }^{2}$ Department of ECE, DIET, Anakapalle, A.P.
}

\begin{abstract}
In this work numerical therapy, called reinitialization which is intrinsically maintained during the level set evolution. This is applied to periodically replace the degraded level set function with a signed distance function because due to the development of irregularities in level set functions. This yields a new type of level set evolution called distance regularized level set evolution (DRLSE). The distance regularization effect eliminates the dictate for reinitialization and thus avoids its induced statistical errors. In dissimilarity to knotty implementations of straight level set formulations, a simpler and more resourceful finite divergence design can be used to execute the DRLSE formulation. DRLSE also allows the employ of more broad and capable initialization of the level set function. In its numerical implementation, somewhat large time steps can be used in the finite difference scheme to reduce the number of iterations, while ensuring sufficient statistical precision. To reveal the efficacy of the DRLSE formulation, we apply it to an edge-based active contour model for image segmentation, and provide an easy narrowband realization to significantly diminish computational cost.
\end{abstract}

Keywords: Reinitialization, image segmentation, DRLSE

\section{Introduction}

The goal of image segmentation is to partition the image plane into meaningful areas, where meaningful typically refers to a separation of areas corresponding to different objects in the observed scene from the area corresponding to the background. A large variety of segmentation algorithms have been proposed over the last few decades. While earlier approaches were often based on a set of rather heuristic processing steps (cf. Perkins, 1980), optimization methods have become established as more principled and transparent methods: Segmentations of a given image are obtained by minimizing appropriate cost functional. Among optimization methods, one can distinguish between spatially discrete and spatially continuous representations. It was only after the work by Osher and Sethia, the level set method became well known and since then has had far-reaching impact in various applications, such as computational geometry, fluid dynamics, image processing, and computer vision. In recent years, a large body of work on geometric active contours, i.e., active contours implemented via level set methods, has been proposed to address a wide range of image segmentation problems in image processing and computer vision [1]. Level set methods were first introduced by Osher and Sethian for capturing moving broadly classified as eitherparametric active contourmodels orgeometric active contourmodels according to their representation and implementation. In particular, the parametric active contours are represented explicitly as parameterized curves in a Lagrangian framework, while the geometric active contours are represented implicitly as level sets of a two-dimensional function that evolves in an Eulerian framework. A desirable advantage of level set methods is that they can represent contours of complex topology and are able to handle topological changes, such as splitting and merging, in a natural and efficient way, which is not allowed in parametric active contour models unless extra indirect procedures are introduced in the implementations. Another desirable feature of level set methods is that numerical computations can be performed on a fixed Cartesian grid without having to parameterize the points on a contour as in parametric active contour models.

This paper proposes a more general variational level set formulation with a distance regularization term and an external energy term that drives the motion of the zero level contour toward desired locations[2]. The distance regularization term is defined with a potential function such that it forces the gradient magnitude of the level set function to one of its minimum points, thereby maintaining a desired shape of the level set function, particularly a signed distance profile near its zero level set. In particular, we provide a double-well potential for the distance regularization term. The level setevolution is derived as a gradient flow that minimizes this energy functional. In the level set evolution, the regularity of the LSF is maintained by a forward-and-backward diffusion derived from the distance regularization term. As a result, the distance regularization completely eliminates the need for reinitialization in a principled way, and avoids the undesirable side effect introduced by the penalty term in our preliminary work[3]. We call the level set evolution in our formulation a span reginitialization level set evolution (SRLSE). To demonstrate the effectiveness of the SRLSE formulation, we apply it to an edge-based active contour model for image segmentation. We provide a simple and efficient narrowband implementation to further improve the computational efficiency. Due to the distance regularization term, the SRLSE can be 
implemented with a simpler and more efficient numerical scheme in both full domain and narrowband implementations than conventional level set formulations. Moreover, relatively large time steps can be used to significantly reduce the number of iterations and computation time, while ensuring sufficient numerical accuracy.

\section{Image Segmentation ( IS )}

In image processing and computer vision applications, the level set method was introduced independently by Caselleset in the context of active contour (or snake) models for image segmentation. Early active contour models are formulated in terms of a dynamic parametric contour

$C(s, t):[0,1] \times[0, \infty) \rightarrow \mathfrak{R}^{2}$ with a spatial parameter $s$ in which parameterizes the points in the contour, and a temporal variable $t \in[0, \infty)$. The curve evolution can be expressed as

$$
\frac{\partial C(s, t)}{\partial t}=F \mathrm{~N}
$$

Where $\mathrm{F} s$ the speed function that controls the motion of the contour and $\mathrm{N}$ is the inward normal vector to the curve C. The curve evolution in (1) in terms of a parameterized contour can be converted to a level set formulation by embedding the dynamic contour $C(s, t)$ as the zero level set of a time dependent LSF $\phi(x, y, t)$ Assuming that the embedding LSF $\phi$ takes negative values inside the zero level contour and positive values outside, the inward normal vector can be expressed as

$$
\mathrm{N}=-\nabla \phi /|\nabla \phi|
$$

where $\nabla$ is the gradient operator.

When acquiring an image, it might be interesting to know what parts of the image do belong to each other. It could be a satellite image, where we wish to quantify and locate different types of vegetation, or a medical MRI image where the doctors are interested to know how much of each tissue type is present, and where it is located. Image segmentation techniques offer a method to perform these tasks, and can be regarded as the process of dividing an image into groups of pixels which from a preset property are connected to each other.

Segmentation techniques locate objects consisting of pixels having something in common. Commonly this means that pixels with almost the same intensity values are grouped together, or pixels with the same colourcode[4]. There are techniques for finding for instance objects with closed contours, convex objects and the boundaries of an object.

Segmentation techniques have not been widely applied, partly because they are time consuming and partly because there are no overall techniques that are suitable for all different types of images. All intensity based segmentation techniques are sensible to the situation that different objects have almost equal intensities[6]. This will often lead to misclassification (=wrongly classified pixels) if the objects from the view of the human eye should not belong to the same class. The curve evolution equation (1) is converted to the following partial differential equation (PDE):

$$
\frac{\partial \phi}{\partial t}=F|\nabla \phi|
$$

A standard method for reinitialization is to solve the following evolution equation to steady state:

$$
\frac{\partial \psi}{\partial t}=\operatorname{sign}(\phi)(1-|\nabla \psi|)
$$

Where $\phi$ is the LSF to be reinitialized, and sign $(\cdot)$ is the sign function. Ideally, the steady state solution of this equation is a signed distance function[4]. This reinitialization method has been widely used in level set methods. Another method for reinitialization is the fast marching algorithm. Although reinitialization as a numerical remedy is able to maintain the regularity of the LSF, it may incorrectly move the zero level set away from the expected position. 


\section{SRLSE of IS (Image Segmentaion)}

In level set methods, a contour (or more generally a hyper surface) of interest is embedded as the zero level set of an LSF. Although the final result of a level set method is the zero level set of the LSF, it is necessary to maintain the LSF in a good condition, so that the level set evolution is stable and the numerical computation is accurate. This requires that the LSF is smooth and not too steep or too flat (at least in a vicinity of its zero level set) during the level set evolution[7]. This condition is well satisfied by signed distance functions for their unique property $|\nabla \phi|=1$ which is referred to as the signed distance property. For the 2-D case as an example, we consider a signed distance function $z=\phi(x, y)$ as a surface. Then, its tangent plane makes an equal angle of $45^{\circ}$ with both the xy-plane and the z-axis which can be easily verified by the signed distance property $|\nabla \phi|=1$. For this desirable property, signed distance functions have been widely used as level set functions in level set methods. In conventional level set formulations, the LSF is typically initialized and periodically reinitialized as a signed distance function. In this section, we propose a level set formulation that has an intrinsic mechanism of maintaining this desirable property of the LSF. The level set reinitialization term $R_{p}(\phi)$ is defined by

$$
R_{p}(\phi) \stackrel{\Delta}{=} \int_{\Omega} p(|\nabla \phi|) d x
$$

where $p$ is a potential (or energy density) function $p:[0, \infty) \rightarrow \mathfrak{R}$

The corresponding level set regularization term $R_{p}(\phi)$ is referred to as a distance regularization term for its role of maintaining the signed distance property of the LSF. A simple and straightforward definition of the potential $p$ for distance regularization is

$$
p=p_{1}(s) \stackrel{\Delta}{=} \frac{1}{2}(s-1)^{2}
$$

which has $s=1$ as the unique minimum point. With this potential $p=p_{1}(s)$ the level set regularization term $R_{p}(\phi)$ can be explicitly expressed as

$$
P(\phi)=\frac{1}{2} \int_{\Omega}(|\nabla \phi|-1)^{2} d x
$$

which characterizes the deviation of $\phi$ from a signed distance function[8].

The distance regularization effect in SRLSE can be seen from the gradient flow of the energy $\mu R_{p}(\phi)$

$$
\frac{\partial \phi}{\partial t}=\mu d i v\left(d_{p}(|\nabla \phi|) \nabla \phi\right)
$$

This flow can be expressed in standard form of a diffusion equation

$$
\frac{\partial \phi}{\partial t}=\operatorname{div}(D \nabla \phi)
$$

This flow can be expressed in standard form of a diffusion equation

$$
\frac{\partial \phi}{\partial t}=\operatorname{div}(D \nabla \phi)
$$

with diffusion rate $D=\mu d_{p}(|\nabla \phi|)$.

We demonstrate the distance regularization effect of SRLSE by simulating the FAB diffusion (6) with the initial function $\phi_{0}$. The binary step function $\phi_{0}$ is defined by[9]

$$
\phi_{0}(x)=\left\{\begin{array}{l}
-c_{0}, i f x \in R_{0} \\
c_{0}, \text { otherwise }
\end{array}\right.
$$

Where $c_{0}>0$ is a constant, and $R_{0}$ is a region in the domain $\Omega$.

The narrowband implementation of the SRLSE consists of the following steps: 
Step 1) Initialization.Initialize an LSF $\phi$ to a function $\phi_{0}$. Then construct the initial narrowband $B_{r}^{0}=U_{(i, j) \in Z^{0}} N_{i, j}^{(r)}$, where $Z^{0}$ is the set of the zero crossing points of $\phi^{0}$.

Step 2) Update the LSF. Update $\phi_{i, j}^{k+1}=\phi_{i, j}^{k}+\tau L\left(\phi_{i, j}^{k}\right)$ on the narrowband $B_{r}^{k}$.

Step 3) Update narrowband. Determine the set of all the zero crossing pixels of $\phi_{i, j}^{k+1}$ on $B_{r}^{k}$, denote by $Z^{k+1}$ . Then, update the narrowband by setting $B_{r}^{k+1}+U_{(i, j) \in Z^{k+1}} N_{i, j}^{(r)}$.

Step 4)Assign values to new pixels on the narrowband. For every point $(i, j)$ in the $B_{r}^{k+1}$ but not in $B_{r}^{k}$, set $\phi_{i, j}^{k+1}$ of $h$ if $\phi_{i, j}^{k+1}>0$, or else set $\phi_{i, j}^{k+1}$ to $-h$, where $h$ is a constant, when can be set to $r+1$ asa default value.

Step 5) Setermine the termination of iteration. If either the zero crossing points stop varying for $m$ consecutive iterations or $k$ exceeds a prescribed maximum number of iterations, then stop the iteration, otherwise, go to step 2 .

\section{Initialization Of Level Set Function}

The SRLSE not only eliminates the need for reinitialization, but also allows the use of more general functions as the initial LSFs[10]. We propose to use a binary step function as the initial LSF, as it can be generated extremely efficiently.

Moreover, the region $R_{0}$ can sometimes be obtained by a simple and efficient preliminary segmentation step, such as thresholding, such that is close to the region to be segmented. Thus only a small number of iterations are needed to move the zero level set from the boundary of $R_{0}$ to the desired object boundary.

\section{Results}

This segment shows the results of the SRLSE model for both synthetic and real images. There are parameters $\lambda, \mu$ and $\alpha$ in this model. By contrast, the level set evolution in the GAC model constantly degrades the LSF, from a nice signed distance function to a function with undesirable irregularities.

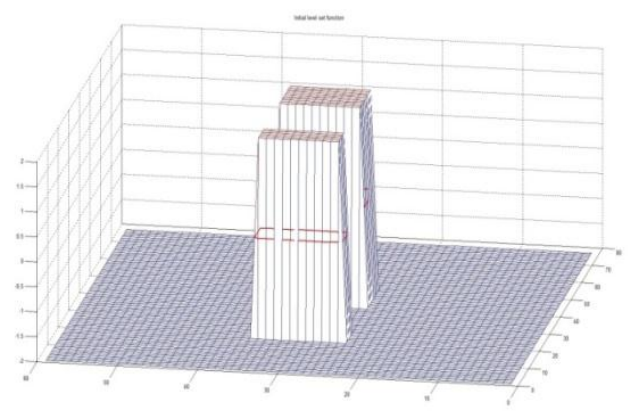

Fig1 Initial level Counter 
Initial zero level contour

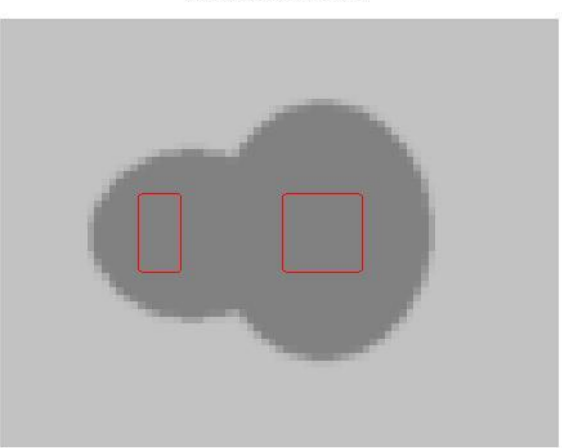

Fig 2. Initial Level Set Function Initial zero level contour

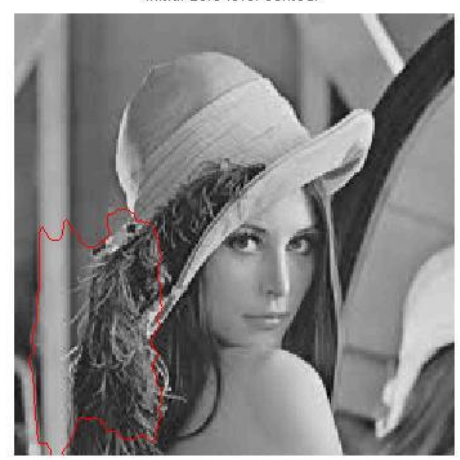

Fig.3 Initial Counter

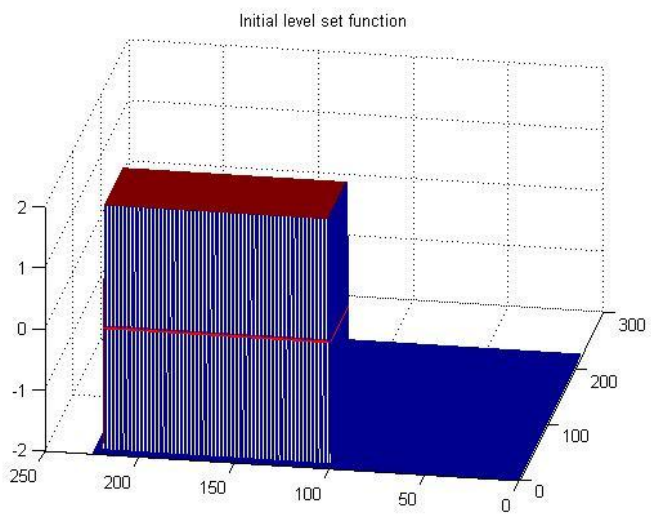

Fig 4 Initial Level Set Function 
Final zero level contour, 110 iterations

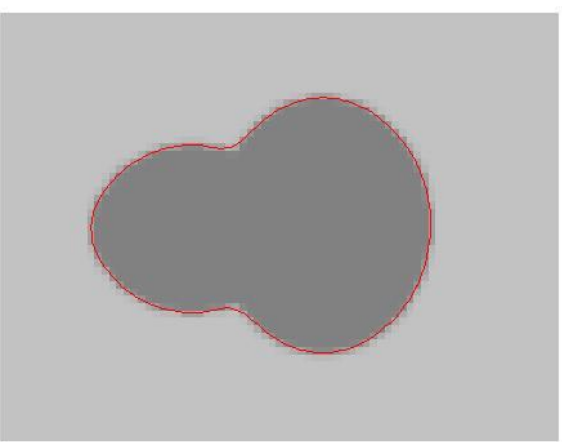

Fig 5. Final Counter

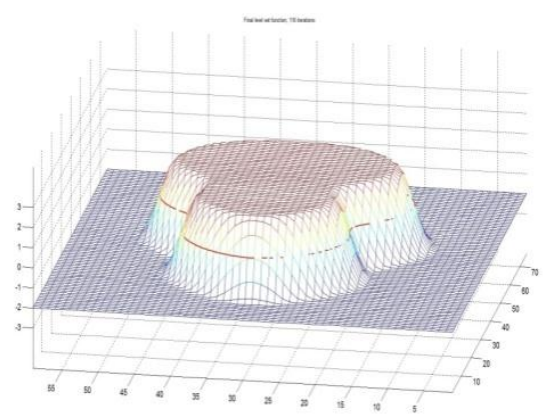

Fig 6. Final LSF

Final zero level contour, 210 iterations

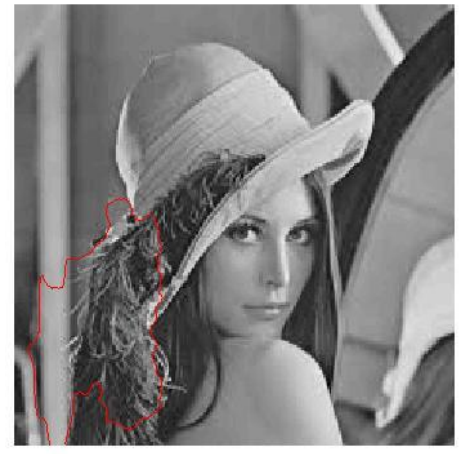

Fig 7. Final Counter

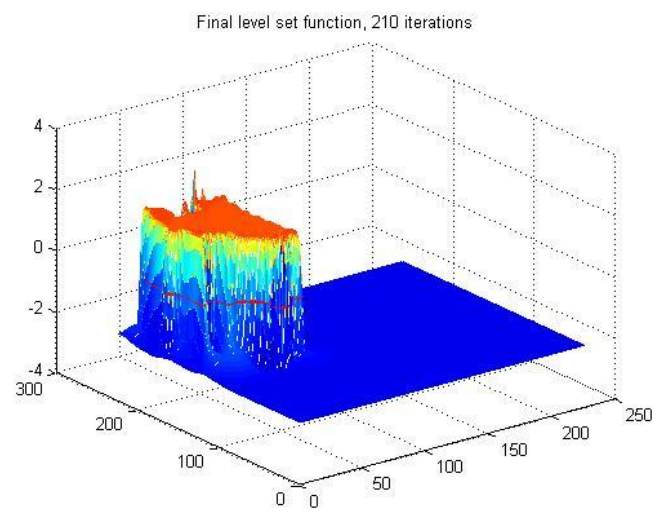

Fig 8. Final Counter 
Table 1 Result analysis

\begin{tabular}{|c|c|c|}
\hline S.No & Figure Number & Counter \\
\hline 1 & 1 & 0 Iterations (Initial) \\
\hline 2 & 5 & 110 Iterations (Final) \\
\hline 3 & 3 & 0 Iterations (Initial) \\
\hline 4 & 7 & 210 Iterations (Final) \\
\hline
\end{tabular}

\section{Conclusion}

We boast on hand a new level set formulation, which weentitle SRLSE. The proposed SRLSE formulation has an built-incapability of maintaining regularity of the level set gathering,particularly the desirable signed distance property in a vicinityof the zero level set, which ensures accurate computation andstable level set evolution. SRLSE can be implemented by asimpler and more efficient numerical scheme than conventionallevel set methods. SRLSE also allows more flexible and proficient initialization than generating a signed distance functionas the preliminary LSF. This vigorous contour model in SRLSE formulation allows the use of relatively large time stepsto drastically diminish iteration facts and computation time, while maintaining sufficient arithmetical accuracy in both fulldomain and narrowband implementations, due to the intrinsicdistance regularization embedded in the level set evolution.Given its first-rate organization and exactness, we expect that the proposeddistance regularized level set evolution will find its utility inmore applications in the area of IS(image segmentation), as wellas added areas wherever level set method has been and could be applied.

\section{References}

[1] S. Kichenassamy, A. Kumar, P. Olver, A. Tannenbaum, and A. Yezzi, "Gradient flows and geometric active contour models," inProc.5th Int. Conf. Comput. Vis., 1995, pp. 810-815.

[2] M. Sussman and E. Fatemi, "An efficient, interface-preserving level set redistancing algorithm and its application to interfacial incompressible fluid flow,"SIAM J. Sci. Comput., vol. 20, no. 4, pp. 1165-1191, Jul. 1999.

[3] G. Aubert and P. Kornprobst, Mathematical Problems in Image Processing: Partial Differential Equations and the Calculus of Variations. New York: Springer-Verlag, 2002.

[4] G. Gilboa, N. Sochen, and Y. Zeevi, "Forward-and-backward diffusion processes for adaptive image enhancement and denoising,'IEEETrans. Image Process., vol. 11, no. 7, pp. 689-703, Jul. 2002.

[5] C. Li, C. Xu, C. Gui, and M. D. Fox, "Level set evolution without re-initialization: A new variationalformulation," inProc. IEEE Conf. Comput. Vis. Pattern Recognit., 2005, vol. 1, pp. 430-436.

[6] M. Sussman and E. Fatemi, "An efficient, interface-preserving level set redistancing algorithm and its application to interfacial incompressible fluid flow,"SIAM J. Sci. Comput., vol. 20, no. 4, pp. 1165-1191, Jul. 1999.

[7] H. Jin, S. Soatto, and A. Yezzi, "Multi-view stereo reconstruction of dense shape and complex appearance,’Int. J. Comput. Vis., vol. 63 , no. 3, pp. 175-189, July 2005.

[8] C. Li, C. Kao, J. C. Gore, and Z. Ding, "Minimization of region-scalable fitting energy for image segmentation," IEEE Trans. Image Process., vol. 17, no. 10, pp. 1940-1949, Oct. 2008.

[9] C. Samson, L. Blanc-Feraud, G. Aubert, and J. Zerubia, "A variational model for image classification and restoration,"IEEE Trans. Pattern Anal. Mach. Intell., vol. 22, no. 5, pp. 460-472, May 2000.

[10] S.-C. Zhu and A. Yuille, "Region competition: Unifying snakes, region growing, and Bayes/MDL for multiband image segmentation,’IEEE Trans. Pattern. Anal. Mach. Intell., vol. 18, no. 9, pp. 884-900, Sep. 1996. 